\title{
LA ENSEÑANZA INICIAL DEL CONCEPTO DE FRACCIÓN POR MAESTROS EN FORMACIÓN
}

\author{
Elena Castro Rodríguez \\ Luis Rico Romero \\ Universidad de Granada \\ Pedro Gómez \\ Universidad de los Andes (Colombia)
}

\begin{abstract}
RESUMEN: En este documento presentamos las indagaciones sobre el conocimiento didáctico del contenido que un grupo de futuros maestros de primaria pusieron en juego al redactar un texto con el cual iniciar a los escolares de primaria en la noción parte-todo de fracción. Para analizar las producciones de los futuros maestros usamos un modelo de análisis didáctico, con categorías propias para los análisis de contenido y de instrucción. Los resultados destacan los contenidos sobre fracciones que los participantes seleccionaron, el modo en que propusieron introducir esos contenidos y el uso que hicieron de las representaciones en sus propuestas.
\end{abstract}

PALABRAS CLAVE: Análisis didáctico, conocimiento didáctico del contenido, formación inicial de maestros, fracciones, relación parte-todo.

\section{INITIAL TEACHING OF THE CONCEPT OF FRACTION BY PRESERVICE PRIMARY SCHOOL TEACHERS}

\begin{abstract}
In this paper we investigate about the pedagogical content knowledge that a group of preservice primary school teachers put into play when composing a text with which to start primary school students in the partwhole notion of fraction. To analyse the productions, we used a model of didactic analysis, through its content and instructional analysis categories. The results highlight the content of fractions that the participants selected, how they proposed to introduce this content and the uses of the representations made in their responses.
\end{abstract}

KEYWORDS: Didactic analysis, fractions, part-whole relationship, pedagogical content knowledge, pre-service teacher education. 


\section{INTRODUCCIÓN}

Una de las formas usuales en que los docentes influyen en la comprensión de conceptos matemáticos por sus estudiantes es mediante las explicaciones que realizan. Estas exposiciones, orales o escritas, son un componente importante en el aprendizaje de los escolares (Charalambous, Hill y Ball, 2011), ya que explicaciones incoherentes, incompletas o poco claras pueden interferir negativamente en el aprendizaje (Weiss y Parsley, 2004).

Diversas investigaciones sobre el conocimiento profesional de los profesores han puesto de manifiesto las dificultades que se les plantean a los profesores en formación cuando realizan exposiciones sobre un contenido matemático (Kinach, 2002; Thanheiser, 2009). Expertos en educación matemática afirman que, a pesar de su relevancia en la enseñanza y aprendizaje de las Matemáticas, lo que conocemos sobre esta cuestión se limita a comparaciones entre las explicaciones ofrecidas por profesores expertos y novatos, así como sobre las dificultades que estos últimos suelen presentar (Charalambous, Hill y Ball, 2011).

Estas dificultades aumentan cuando se trata de contenidos de carácter problemático para los maestros, como es el tema de fracciones (Ball, 1990; Gairin, 2001; Newton, 2008; Simon, 1993). Entre las distintas investigaciones realizadas sobre el conocimiento didáctico de las fracciones (Charalambous, Hill y Ball, 2011; Domoney, 2001; Fuller, 1996), encontramos estudios centrados en las operaciones con fracciones (Li y Kulm, 2008; Isiksal y Cakiroglu, 2011) o en la equivalencia de fracciones (Chick, 2003; Marks, 1990). Todos ellos resaltan las carencias que los maestros en formación presentan en sus conocimientos sobre la relación multiplicativa parte-todo y las fracciones y, por consiguiente, en las implicaciones para su enseñanza. Además, Charalambous, Hill y Ball (2011) y Li y Kulm (2008) afirman que la falta de conocimiento del contenido sobre fracciones, por parte de los profesores participantes en sus estudios, puede incidir en los niveles mostrados en su conocimiento didáctico de este contenido.

Esta cuestión y su escaso tratamiento en la literatura nos condujeron a centrarnos en un aspecto del conocimiento didáctico de los futuros maestros: su capacidad para introducir un contenido matemático con un propósito didáctico. El conocimiento didáctico del contenido, en el sentido de Hill, Ball, y Schilling (2008), implica estos y otros aspectos de las decisiones que el profesor toma en su proceso de planificación de la enseñanza.

\section{ESTUDiO DEL CONOCIMIENTO DIDÁCTICO DEL CONTENIDO}

En el caso de los profesores, el conocimiento profesional involucra el conocimiento relativo a la práctica lectiva en el salón de clases y en otros papeles profesionales, tales como la tutoría a los alumnos, la participación en actividades y proyectos de la escuela, la interacción con miembros de la comunidad y el trabajo en asociaciones profesionales (Climent y Carrillo, 2002).

La naturaleza, caracterización y complejidad del conocimiento del profesor de matemáticas han sido descritos por investigadores como Shulman (1986), Hill, 
Ball y Schilling (2008), Bromme (1994), Ponte y Chapman (2008), entre otros, todos ellos consideran que el conocimiento profesional del maestro tiene diversas dimensiones.

Basándose en el trabajo de Shulman (1986), Hill, Ball y Schilling (2008) sostienen que el conocimiento del profesor de matemáticas presenta dos dimensiones denominadas Subject Matter Knowledge y Pedagogical Content Knowledge, ambas subdivididas en otros tipos. Desde esta perspectiva, proponen las siguientes componentes relativas al Pedagogical Content Knowledge: Knowledge of Content and Student, Knowledge of Content and Teaching y Knowledge of Curriculum. El primero de ellos definido como "el conocimiento del contenido vinculado con el conocimiento de cómo los estudiantes piensan, saben, o aprenden un contenido particular" (Hill, Ball y Schilling, 2008: 375), es considerado uno de los componentes primarios de la noción Pedagogical Content Knowledge expuesto por Shulman (1986). En segundo lugar, Knowledge of Content and Teaching, engloba conocer cómo abordar la enseñanza de modo tal que ésta permita a los estudiantes superar errores y concepciones inadecuadas. Además, este conocimiento implica cuestiones relacionadas con la selección de tareas, técnicas de gestión de clase y materiales o recursos para el tratamiento de cierto contenido. Por último, Knowledge of Curriculum, se refiere al conocimiento sobre qué contenidos deben aprender los estudiantes y la orientación que deben tomar esos contenidos en el aprendizaje e incluye los materiales curriculares de los que hace uso el profesor y el conocimiento de los contenidos y planes de estudios.

En nuestro trabajo entendemos el conocimiento didáctico del contenido como:

"el conjunto de conocimientos y habilidades que los facultan para abordar el análisis de una estructura matemática con el propósito de producir y justificar una planificación. Desde esta perspectiva, el conocimiento didáctico de un grupo de futuros profesores se configura alrededor de un conjunto estructurado de capacidades que caracterizan su competencia planificación." (Gómez, 2007: 122).

Entre los distintas investigaciones realizadas sobre el conocimiento didáctico del contenido, encontramos diversos estudios sobre fracciones (Charalambous, Hill y Ball, 2011; Domoney, 2001; Fuller, 1996), centrados en las operaciones con fracciones (Li y Kulm, 2008; Isiksal y Cakiroglu, 2011; Charalambous, Hill y Ball, 2011) o en la equivalencia de fracciones (Chick, 2003; Marks, 1990). Algunos de estos estudios (D'Ambrosio y Mendoça, 1992; Domoney, 2001; Marks, 1990) sugieren que es necesario el diseño de instrumentos adecuados para el estudio del conocimiento didáctico del contenido, pues los empleados en estudios previos presentaron algunos inconvenientes. Gómez y Gutiérrez-Gutiérrez (2014), analizaron el conocimiento didáctico en el subdominio de números que manifestaron los futuros profesores españoles en el estudio del conocimiento del profesor en formación TEDS-M (Teacher Education Study in Mathematics). Estos autores subrayaron que una limitación en el estudio TEDS-M fue la elección de una herramienta idónea para la evaluación, y por consiguiente, la obtención de resultados propios sobre el conocimiento didáctico del contenido.

Para intentar solventar las limitaciones anteriores, en este trabajo consideramos este tipo de conocimiento desde una perspectiva particular sobre la práctica docente. 
En la práctica docente, el profesor puede abordar la planificación de la enseñanza de un contenido matemático poniendo en juego su conocimiento didáctico al seleccionar conceptos y procedimientos y formular tareas, secuenciarlas y proponer actividades (Gómez y González, 2013). En esa planificación, el profesor necesita de comprensión y dominio del contenido para tomar decisiones sobre qué conceptos, procedimientos, representaciones y fenómenos considera relevantes; qué expectativas, limitaciones y oportunidades de aprendizaje considera que se deben lograr, abordar y diseñar, respectivamente; y qué tareas debe proponer a los estudiantes para que ellos tengan la oportunidad de lograr esas expectativas de aprendizaje y superar esas limitaciones de aprendizaje. El conocimiento didáctico del contenido implica estos y otros aspectos de las decisiones que el profesor toma en su proceso de planificación.

Siguiendo esta línea, en este trabajo propusimos a los maestros que redactaran una explicación introductoria al concepto de fracción. Para ello, diseñamos una serie de ilustraciones que presentan los datos básicos de una relación parte-todo multiplicativa y pedimos a los maestros que redactaran una explicación con la que introducir la noción de fracción a partir de las imágenes presentadas. En la tarea propuesta, los participantes debían realizar una breve planificación sobre la enseñanza de las fracciones. Para ello, se basaron en los datos propuestos en las ilustraciones, además de poner en juego su conocimiento didáctico del contenido. Para el análisis de las producciones consideramos útil la noción de análisis didáctico, el cual tomamos como base para organizar los aspectos del conocimiento didáctico de nuestro interés.

\section{ANÁLISIS DIDÁCTICO}

Por análisis didáctico, entendemos un procedimiento que, de manera ideal, debería realizar un profesor de Matemáticas para "diseñar, llevar a la práctica y evaluar actividades de enseñanza y aprendizaje" (Rico y Fernández-Cano, 2013). Al caracterizar, de manera ideal, el proceso de planificación del profesor, el modelo del análisis didáctico permite establecer aquellos aspectos del conocimiento didáctico del profesor que son relevantes para ese propósito. Esos aspectos se organizan en cuatro tipos de análisis: un análisis de contenido, un análisis cognitivo, un análisis de instrucción y un análisis de actuación. Estos análisis, que permiten estructurar el conocimiento didáctico, han sido trabajados y utilizados en diversos estudios e investigaciones (Gómez, 2006; Lupiáñez y Rico, 2008; Valverde, 2012).

Nos centramos en el conocimiento didáctico del contenido que los maestros de primaria en formación inicial ponen en juego al redactar un texto con el cual iniciar a los escolares de primaria en la noción de fracción mediante una relación partetodo. Para ello, abordamos el análisis de las redacciones realizadas mediante su análisis de contenido y su análisis de instrucción, cada uno de los cuales se estructura mediante sus propias componentes. A continuación, describimos estos dos análisis y sus componentes, aplicados a la noción parte-todo de fracción.

\section{Análisis de contenido}

El análisis de contenido se centra en analizar, describir y establecer los diferentes significados que tienen los conceptos y procedimientos que son objeto de ense- 
ñanza y aprendizaje y que el profesor considera relevantes a efectos de su planificación para la instrucción. Su propósito es la descripción de la estructura matemática que se analiza, desde la perspectiva de las Matemáticas escolares. Nuestro estudio articula el análisis de contenido según tres componentes: la estructura conceptual, los sistemas de representación y la fenomenología.

\section{Estructura conceptual}

La estructura conceptual incluye conceptos, propiedades, proposiciones y relaciones entre los conceptos, que se derivan de un contenido matemático (Gómez, 2006). En el caso del concepto parte-todo de fracción consideramos los siguientes componentes básicos en su estructura: (a) el todo -T- que tomamos como punto de partida; (b) cada una de las $\mathrm{n}$ partes iguales en que se divide el todo - $\mathrm{P}-$; (c) la relación $-R(P, T)=\frac{1}{n}-$ que expresa la relación entre una de las partes iguales $P$ y el todo $\mathrm{T}$; y (d) el complementario $\mathrm{C}$ de la parte $\mathrm{P}-$.

\section{Representaciones}

Los conceptos se muestran a través de diferentes tipos de símbolos escritos, gráficos, imágenes o el lenguaje hablado, y cada uno constituye una representación (externa) del concepto en cuestión (Hiebert y Carpenter, 1992). Las fracciones como relación parte-todo pueden ser representadas de múltiples formas como son: representaciones verbales, gráficas, numéricas o simbólicas.

\section{Fenomenología}

La fenomenología muestra los sentidos de los cuales proceden y con los cuales se usan los conceptos, sentidos que los vinculan con los mundos natural, cultural, social y científico; también muestra su conexión con las estructuras matemáticas En el concepto parte-todo de fracción encontramos diversos sentidos, como son: división, reparto, medida o reconstrucción de la unidad.

\section{Análisis de instrucción}

El análisis de instrucción, aborda las decisiones del docente relacionadas con la actividad de enseñanza, como la selección y el diseño de tareas o la organización de sesiones de clase. Dado que estudiamos cómo los maestros en formación realizan una introducción del concepto de fracción a un grupo de escolares, nos centramos en una componente fundamental del análisis de instrucción: la explicación del profesor. Dentro de esta componente identificamos dos categorías para el análisis de las respuestas: modos de introducir los contenidos y usos de las representaciones.

La primera aborda los modos en que los participantes introducen en su explicación los contenidos seleccionados, que identificamos como modo instrumental, modo funcional y modo narrativo. En el modo instrumental, la redacción no incluye situaciones ni problemas contextualizados que puedan ayudar en la comprensión de los contenidos. En el modo funcional se aborda el contenido a través de situaciones contextualizadas y presentando demandas cognitivas al escolar, la mayor parte de las veces a través de la resolución de problemas. En el modo narrativo, al 
igual que en el caso anterior, se introducen los contenidos a través de un texto que modeliza una situación real pero no se incluye ninguna demanda cognitiva.

La segunda componente, uso de representaciones, considera la función que tienen las ilustraciones dadas en la tarea, usos que son ilustrativos, explicativos y aclarativos. El primer tipo de uso agrupa aquellas respuestas en las que las imágenes sólo tienen una función ilustrativa: se usan únicamente para acompañar al texto. La explicación contenida en el texto es lo suficientemente completa para que sea comprensible, aun si se suprimen las imágenes. En el segundo uso, las imágenes tienen una función explicativa. Son respuestas escuetas, con escaso texto que no presenta ningún aspecto matemático; simplemente son expresiones introductorias o frases que enlazan unas imágenes con otras. La explicación la hace la serie de imágenes por lo que, si éstas se suprimen, el texto deja de tener sentido, ya que por si sólo no explica ningún contenido. El último de los tipos de usos se encuentra en una situación intermedia a las dos anteriores. Las imágenes tienen una función aclarativa: forman parte de la explicación y la mejoran. El texto presenta información matemática pero por sí sólo no tiene sentido ni forma la explicación completa. La particularidad de este uso es que la combinación de texto e imágenes constituyen la explicación, en la que las ilustraciones ejemplifican, dan más detalles, aclaran o muestran nuevos contenidos.

\section{OBjetivo DE INVESTIGACIÓN}

En este trabajo nos propusimos como objetivo explorar y describir el conocimiento didáctico del contenido que ponen en juego los maestros en formación inicial al introducir el concepto de fracción mediante una relación parte-todo.

\section{MÉTODO}

\section{Sujetos}

Los participantes de este estudio fueron 82 maestros en formación inicial que cursaban los estudios universitarios del Grado en Educación Primaria durante el curso académico 2011-2012. Los sujetos eran estudiantes del segundo curso de dicha titulación, matriculados en tres grupos distintos de la asignatura Enseñanza y Aprendizaje de las Matemáticas en Educación Primaria.

\section{Instrumento}

El instrumento que utilizamos en este estudio para incentivar el trabajo de los maestros en formación, y posteriormente recoger información, consta de diversas series de imágenes, cada una con tres tarjetas; las imágenes muestran objetos que son usuales en la introducción inicial de las fracciones. En la serie de tarjetas A, que denominaremos A1, A2 y A3, incluimos ilustraciones de objetos que ejemplifican distintas magnitudes que dan lugar a las fracciones unitarias $\frac{1}{2}, \frac{1}{3}$ y $\frac{1}{4}$. Cada una de las ilustraciones presentes en las tarjetas muestran diferentes elementos básicos de una relación parte-todo multiplicativa: el todo o totalidad $(T)$, las partes $(P)$, y la relación entre una de las partes y el todo $P=\frac{1}{n} T$. 


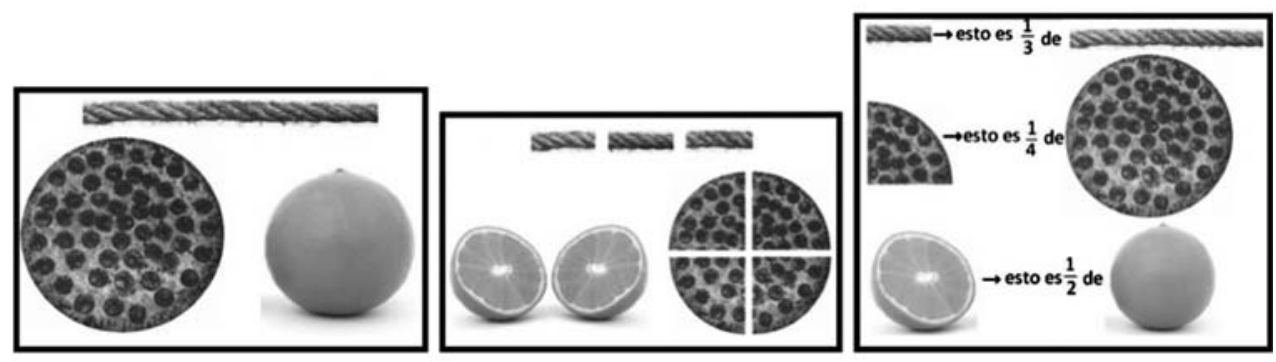

Figura 1. Tarjeta $A 1, A 2$ y $A 3$

Las primeras ilustraciones de la tarjeta A1 (figura 1) muestran los objetos enteros, que en cada caso representan el todo del que se parte, con una, dos o tres dimensiones. En la tarjeta A2 se incluyen los objetos iniciales divididos en partes iguales. Por último, en las ilustraciones de la tarjeta A3 se muestra la relación de una de esas partes con el todo del que procede. Además de estas tarjetas, se proporcionó una ficha de trabajo que incluía las instrucciones para que los sujetos, de manera individual, realizaran la tarea. Esta ficha incluye el siguiente enunciado de la tarea propuesta:

Las tres tarjetas que aparecen a continuación pueden usarse para ilustrar el concepto de fracción. Se desea elaborar un material para iniciar a los alumnos de primaria en las fracciones. Establece el orden en que las tarjetas deben aparecer y redacta el texto que debe ir antes y después de cada tarjeta (como si fuese un libro de texto para primaria).

Subrayamos la idea de que el grupo de escolares de primaria al que va dirigido el material es un grupo hipotético. No lo condicionamos a una edad ni a un nivel determinados; sólo se subraya la idea de que la actividad consiste en una introducción o iniciación al concepto de fracción.

Las ilustraciones fueron impresas como pegatinas para que pudiesen ser manejadas e insertadas libremente a criterio del estudiante durante el proceso de elaboración de la narración. La finalidad de la tarea es inducir a los sujetos a una situación docente, simulando las imágenes de un libro de texto escolar o de una ficha de trabajo. Para ello, dimos las ilustraciones de ese supuesto libro o ficha, pedimos a los maestros que las ordenaran y que escribieran un texto que acompañara y que explicara cada imagen.

\section{Procedimiento}

Para detectar y solventar posibles errores de interpretación y para que los maestros en formación se familiarizasen con la actividad, se realizó una prueba piloto, dos semanas antes, en la que los sujetos debían realizar una tarea similar sobre el concepto de multiplicación. La prueba piloto mostró que la actividad era clara, por lo que el procedimiento seguido y el tipo de material proporcionado para la actividad final fue similar al utilizado en la prueba piloto.

Durante el desarrollo de una sesión de clase, se entregó a cada uno de los sujetos una ficha y una de las series de tarjetas. Una vez distribuido todo el material, se 
les explicó cómo realizar la actividad y se respondió a las dudas que surgieron. Todos los estudiantes finalizaron la actividad en un tiempo máximo de media hora.

\section{Resultados}

Se obtuvieron un total 82 respuestas de las cuales presentamos un ejemplo a continuación.

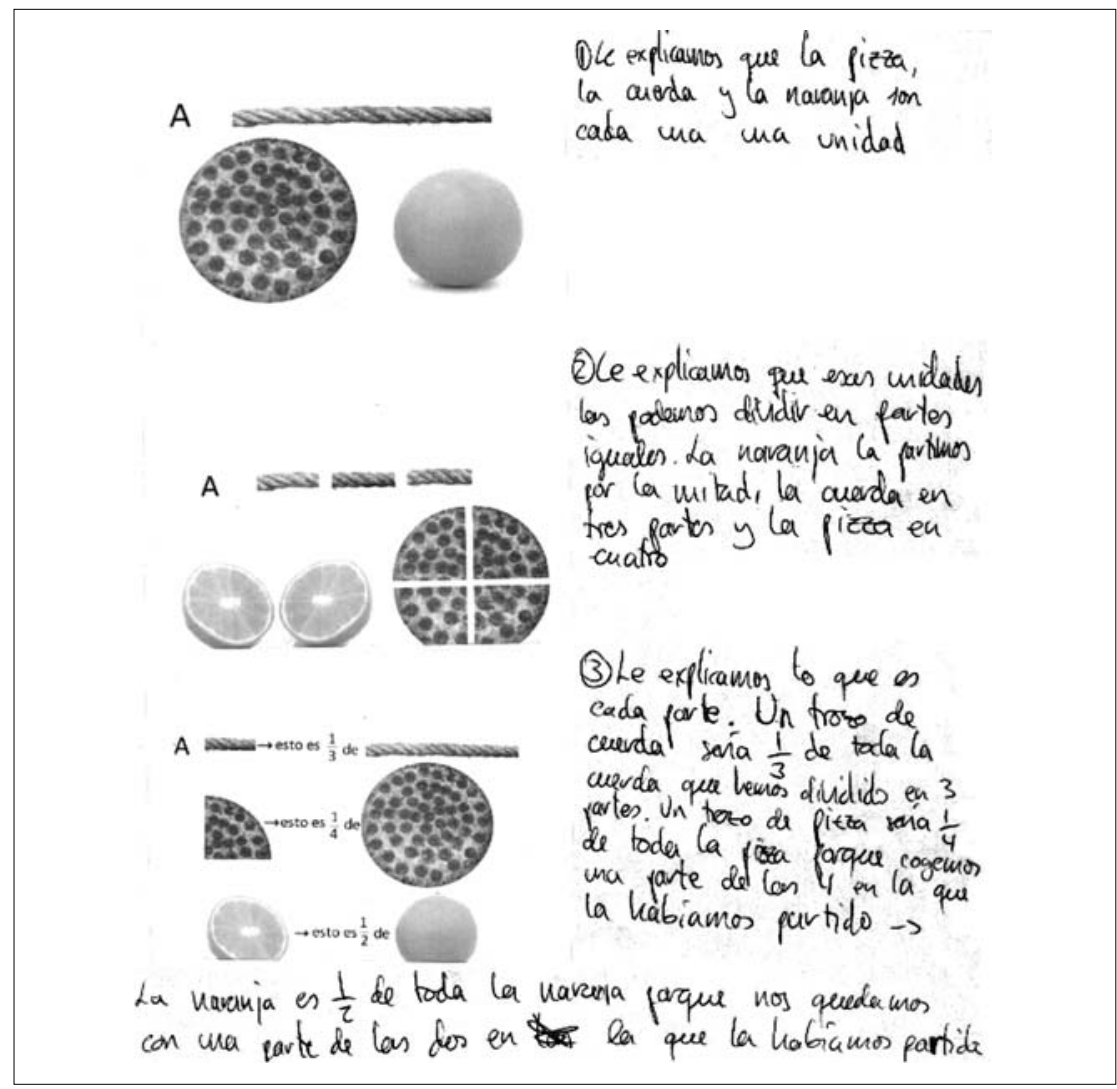

Figura 2. Ejemplo de narración realizada por un estudiante para maestro

En el análisis de los datos utilizamos técnicas cualitativas, cuyo objetivo es organizar y caracterizar las producciones a través del sistema de categorías. Para codificar y categorizar las respuestas, nos basamos en las componentes del análisis de contenido (estructura conceptual, representaciones y fenomenología) y análisis de instrucción (modos de introducir los contenidos y función de las ilustraciones). Estas componentes generales se concretan a través de nuestro marco conceptual en subcategorías más específicas. Además, tras una primera revisión de todas las respuestas, fue necesario ampliar las subcategorías, pues de los datos surgieron otras nuevas no contempladas. Un ejemplo de codificación para la respuesta de la figura 2, se incluye en la tabla 1. 
Tabla 1. Ejemplo de codificación de los datos

\begin{tabular}{|c|c|c|}
\hline \multicolumn{3}{|c|}{ Análisis de contenido } \\
\hline Estructura conceptual & Representaciones & Fenomenología \\
\hline $\begin{array}{l}\text { Todo (pizza, cuerda, naranja); unidad; } \\
\text { partes iguales; parte; } P=\frac{1}{n} T\end{array}$ & $\begin{array}{l}\text { Verbal (mitad) y } \\
\text { numérica }\end{array}$ & $\begin{array}{l}\text { La fracción surge de una división en } \\
\text { partes iguales y selección de algunas }\end{array}$ \\
\hline \multicolumn{3}{|c|}{ Análisis de instrucción } \\
\hline \multicolumn{2}{|c|}{ Modo de introducir contenidos } & Uso de representaciones \\
\hline \multicolumn{2}{|l|}{ Instrumental } & Ilustrativa \\
\hline
\end{tabular}

Los resultados obtenidos se presentan en dos secciones que corresponden a los dos análisis considerados, de contenido y de instrucción.

\section{Datos sobre el análisis de contenido}

En una instrucción inicial sobre el concepto parte-todo de fracción no es posible poner en juego todos los elementos del contenido del tema. Por ello, al realizar una explicación, los sujetos seleccionan aquellas componentes del concepto que conocen y/o eligen los que les parecen más adecuados para comunicar los nuevos conocimientos e iniciar y guiar el aprendizaje de los escolares. Estos conocimientos manifestados fueron codificados según cada una de las componentes del análisis del contenido: estructura conceptual, fenomenología y representaciones. Estas tres componentes permiten identificar, analizar e interpretar las producciones realizadas por los maestros en formación en términos de aquellos aspectos del contenido que seleccionan para realizar una introducción al concepto de fracción.

\section{Datos sobre la estructura conceptual}

En nuestro análisis identificamos conceptos y procedimientos distintos de los presentes en las ilustraciones que los sujetos añadieron como conocimiento adicional en sus respuestas. Como vemos en el ejemplo de la figura 2, los participantes introdujeron en su narración la explicación del concepto de unidad. Algunos de estos conceptos no se presentaron en el marco teórico como elementos fundamentales de la estructura conceptual (todo, parte, relación y complementario), por lo que se añadieron como nuevos valores para esta categoría. Los elementos, todo, parte y relación, fueron incluidos en casi la totalidad de respuestas, por el contrario el elemento complementario se incluyó sólo en el 24\% de respuestas. La tabla 2 recoge aquellos valores para la categoría estructura conceptual que no se consideraron en el marco teórico. 
Tabla 2. Conceptos y procedimientos añadidos en las respuestas

\begin{tabular}{|l|l|c|}
\hline $\begin{array}{l}\text { Concepto o } \\
\text { procedimiento }\end{array}$ & Ejemplo de respuesta & $\begin{array}{c}\text { Porcentaje } \\
\mathbf{N = 8 2}\end{array}$ \\
\hline $\begin{array}{l}\text { Concepto de } \\
\text { numerador y } \\
\text { denominador }\end{array}$ & $\begin{array}{l}\text { "...en una fracción, el número o parte que cogemos del total se } \\
\text { denomina numerador y el número en que dividimos el total y } \\
\text { que se posiciona debajo es el denominador" }\end{array}$ & $34 \%$ \\
\hline $\begin{array}{l}\text { Concepto de } \\
\text { fracción entera }\end{array}$ & $\begin{array}{l}\text { “..como la cuerda la hemos dividido en 3 partes, la parte } \\
\text { entera y completa sería } \frac{3}{3} \text {, ya que 3 dividido entre 3 es 1 que } \\
\text { es la parte entera..." }\end{array}$ & $17 \%$ \\
\hline $\begin{array}{l}\text { Concepto de } \\
\text { unidad }\end{array}$ & $\begin{array}{l}\text { "para la explicación de las fracciones, hemos cogido tres } \\
\text { objetos: pizza, naranja y una cuerda. Estos objetos representan } \\
\text { la unidad, es decir 1" }\end{array}$ & $14 \%$ \\
\hline $\begin{array}{l}\text { Suma o resta } \\
\text { de fracciones }\end{array}$ & $\begin{array}{l}\text { "cada trozo equivale a } \frac{1}{3} \text { y tenemos 3 trozos, } \frac{1}{3}+\frac{1}{3}+\frac{1}{3}= \\
\text { cuerda completa"; "nos comemos una porción } \frac{1}{4}, \text { al restarle } \\
\frac{1}{4} \text { a los } \frac{4}{4} \text { nos quedan } \frac{3}{4} \text { " }\end{array}$ \\
\hline
\end{tabular}

Como se aprecia en la tabla 2, el conocimiento adicional más común consiste en identificar el significado del numerador y denominador con los elementos de la estructura conceptual en un proceso de división en partes de un objeto o en un proceso de reparto (34\%). Otros dos contenidos que se incluyen con un porcentaje similar en las respuestas son el concepto de fracción entera y su relación con el todo dividido en partes (17\%) y el concepto de unidad y su identificación con el todo u objeto inicial (14\%). Por último, en algunas respuestas se introducen otros aspectos procedimentales como la suma y resta de fracciones, aunque en ningún caso se incluye el algoritmo de estas operaciones.

\section{Datos sobre fenomenología}

A pesar de que las ilustraciones inducen un proceso de división en partes, en sus respuestas los sujetos introducen otros sentidos distintos: repartir, medir y reconstruir la unidad dada una fracción. En este caso, las respuestas no incluyen sentidos distintos a los considerados en el marco conceptual, por lo que no se ampliaron los valores para esta categoría.

Tabla 3. Sentidos presentes en las respuestas

\begin{tabular}{|l|c|}
\hline & Porcentaje \\
\hline $\begin{array}{l}\text { Las fracciones surgen de una división en partes de un objeto y la selección de } \\
\text { algunas de ellas }\end{array}$ & $37 \%$ \\
\hline $\begin{array}{l}\text { Las fracciones surgen de una división en partes de un objeto y la medida de } \\
\text { una de las partes }\end{array}$ & $20 \%$ \\
\hline Las fracciones surgen de una división en partes de un objeto & $20 \%$ \\
\hline Las fracciones surgen de un proceso de división y reparto & $10 \%$ \\
\hline Las partes se recomponen dando lugar a la unidad & $7 \%$ \\
\hline Las fracciones surgen de un reparto & $6 \%$ \\
\hline
\end{tabular}


Como se observa en la tabla 3, los sentidos se presentan en las respuestas de manera única, como en el ejemplo de la figura 2, o combinando los sentidos de división con medida o reparto.

\section{Datos sobre representaciones}

Puesto que la tarea propuesta a los sujetos contiene ilustraciones con elementos gráficos y numéricos, solamente en 5 casos se incluyeron representaciones gráficas o simbólicas distintas de las dadas en las ilustraciones: una simbólica, tres gráficas, y una simbólica y gráfica.
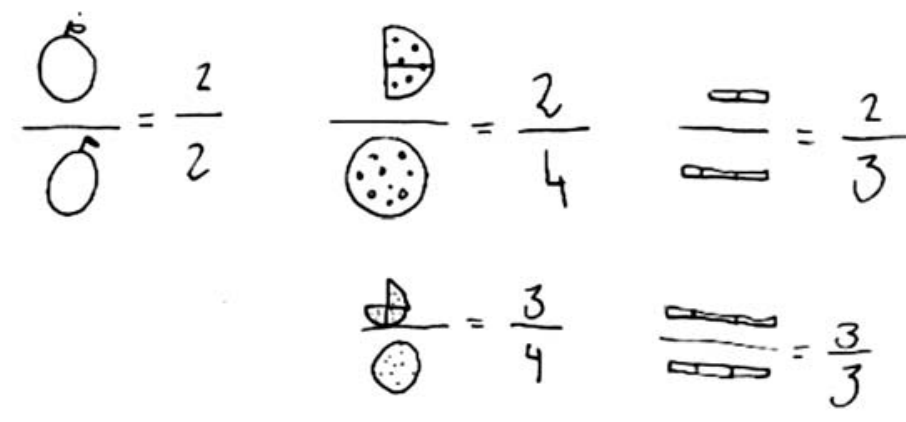

Figura 3. Ejemplo de representación gráfica presente en una respuesta

Estas nuevas representaciones surgen para plantear nuevos ejemplos (figura 3), o reforzar la explicación de los ya presentes en las ilustraciones propuestas. No se detectan representaciones más abstractas de las mismas nociones, que muestren o aborden el concepto en estudio.

\section{Datos sobre el análisis de Instrucción}

Estos datos recogen los aspectos relativos a la explicación propuesta. En esta categoría, se identifican dos valores distintos: los modos en que los participantes introducen los contenidos seleccionados y el uso de las representaciones.

\section{Modos de introducir los contenidos}

Durante su redacción, los participantes, además de seleccionar aquellas componentes del concepto que conocen y consideran más adecuadas, utilizan diferentes modos de presentar los contenidos. Organizamos las producciones según tres modalidades: instrumental, narrativa y funcional.

El modo instrumental es predominante en las respuestas (73\%). Un ejemplo de respuesta para este estilo se encuentra en la figura 2.

El modo funcional tiene una presencia escasa en las respuestas (13\%). En la siguiente respuesta podemos verlo reflejado. 
- (Tarjeta A1) Una familia de 4 personas, quiere repartirse una pizza pero no sabe cómo.

- (Tarjeta A2) Como son 4 personas, dividen en 4 partes quedando así $\frac{1}{4}, \frac{1}{4}, \frac{1}{4}$, $\frac{1}{4}$, todo sumando da $\frac{4}{4}$.

- (Tarjeta A3) Cada uno pues, se come $\frac{1}{4}$ de pizza. El hijo se ha comido ya $\frac{1}{4}$ de pizza así que quedan $\frac{3}{4}$ de pizza.

En el modo narrativo tiene una presencia similar al caso anterior (14\%). Una respuesta que se corresponde con este estilo es la siguiente.

Hoy vamos a aprender lo que es una fracción, nos basamos en un ejemplo sencillo para ello.

- (Tarjeta A1) Como vemos en la figura 1 la pizza está entera, si queremos comerla deberíamos de partirla. Como estamos 4 amigos deberíamos de partirla, un trozo para cada uno.

- (Tarjeta A2) Como somos buenos amigos los trozos serán iguales para todos. Partiremos nuestra pizza y nos quedará como en la figura 2. Si tuviéramos que decir a cuánto nos ha tocado cada uno y cuánto al resto ¿cómo lo haremos?

- (Tarjeta A3) ¡Exacto! Con fracciones. Si la pizza la partimos en 4 trozos y nos quedamos con un trozo lo que les toca a los demás es 3/4 como aparece en la figura 3. Una fracción es una parte de la unidad. La unidad es la pizza, las porciones las partes en las que dividimos, y lo que nos corresponde (nuestra porción) es una fracción.

Al cruzar esta categoría para el análisis de la explicación con la categoría fenomenología para el contenido, se observó que en los modos narrativo y funcional, la mayoría de los participantes utilizaron el sentido de reparto, mientras que en el enfoque instrumental lo hicieron mediante el sentido de división.

\section{Datos sobre el uso de representaciones}

Puesto que la tarea propuesta obliga a los participantes en el estudio a usar unas determinadas ilustraciones, ellos les otorgaron una función determinada dentro de su narración. Estas funciones son ilustrativa, explicativa o aclarativa.

La primera de esas funciones, en la que las imágenes ilustran la explicación pero no añaden o completan con nuevo conocimiento, es usada por un $43 \%$ de los participantes. El ejemplo de respuesta dado en la figura 2 se encuentra dentro de esta categoría.

La segunda, aquella en la que las imágenes tienen una función explicativa. es la que obtiene menor frecuencia (14\%). Un ejemplo de respuesta para este valor de la categoría es: "El primer lugar presentamos a los alumnos los elementos (tarjeta A1), a continuación que es lo que queremos conseguir (tarjeta A2), por último les mostramos la equivalencia de cada porción en referencia al todo (tarjeta A3)".

El último valor de esta categoría, la función aclarativa, está presente en un 43\% de las producciones. Un fragmento de respuesta, que pertenece a este caso se encuentra en la figura 4. 


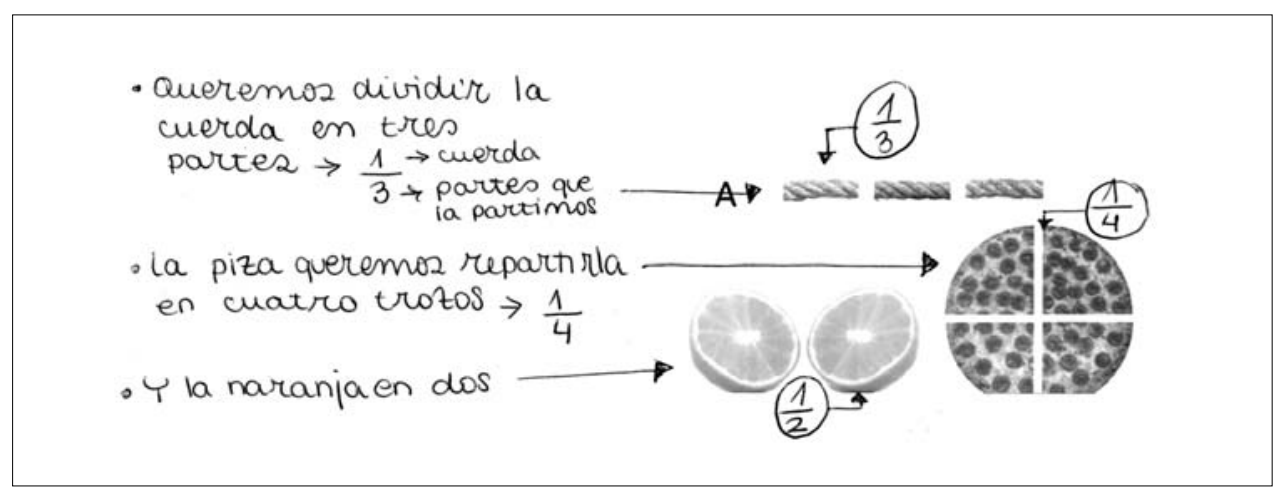

Figura 4. Ejemplo de ilustración con función aclarativa

En este ejemplo las imágenes y el texto se compaginan y forman parte de la explicación, relacionándose con ella a través de flechas.

\section{DISCUSIÓN Y CONCLUSIONES}

El objetivo de este estudio fue caracterizar el conocimiento didáctico del contenido, desde la perspectiva del análisis de contenido y el análisis de instrucción, que un grupo de maestros en formación presentó cuando abordó una explicación para introducir el concepto de fracción. De la información recogida destacamos que, sin ninguna dificultad, los sujetos fueron capaces de ponerse en el papel de docentes y manifestar su conocimiento didáctico del contenido a través de sus respuestas. Además, manifestaron un conocimiento didáctico del contenido coherente en sus explicaciones, en el que se destaca la diversidad de modos en que los sujetos reconstruyen, adecuan o reestructuran el contenido para hacerlo comprensible a los escolares. A pesar de que los sujetos reconocieron los elementos básicos de las fracciones y los pusieron de manifiesto en sus explicaciones junto con otros contenidos, consideramos que una carencia a destacar en este conocimiento es la limitada planificación de la secuenciación de los contenidos atendiendo a su nivel dificultad.

Un logro de este estudio radica en que, a través de un instrumento aparentemente sencillo, nos pudimos aproximar a este tipo de conocimiento salvando las dificultades de otros estudios (Charalambous, Hill y Ball, 2011; Li y Kulm, 2008), en los que las carencias en el conocimiento del contenido sobre fracciones incidió en sus resultados. A pesar de que otros estudios utilizan ítems similares "¿Cómo explicarías las fracciones a alguien que no sabe lo que son?" (Domoney, 2001), la información obtenida no logró las expectativas esperadas, ya que las respuestas fueron escuetas debido al modo de plantear el ítem y el contexto en el que fue propuesto. Nuestro modo de formular la actividad y el uso de ilustraciones a través de tarjetas, dio lugar a una mayor riqueza de respuestas y resultados. Además, el contexto de la asignatura hizo que la dinámica de trabajo estuviese orientada hacia la enseñanza y aprendizaje de las Matemáticas. 


\section{AGRADECIMIENTOS}

Este trabajo ha sido realizado con la ayuda y financiación del proyecto "Procesos de Aprendizaje del Profesor de Matemáticas en Formación" (EDU2012-33030) del Plan Nacional I+D+I (MICIN).

\section{REFERENCIAS BIBLIOGRÁFICAS}

BALL, D. (1990). Preservice elementary and secondary teachers' understanding of division. Journal for Research in Mathematics Education, 21 (2), 132-144.

BROMME, R. (1994). Beyond subject matter: "A psychological topology of teachers' professional knowledge". En R. Biehler, R. Scholz, R. StraBer y B. Winkelman (Eds.), Didactics of Mathematics as a Scientific Discipline (pp. 73-88). Dordrecht: Kluwer Academic.

CHARALAMBOUS, C. Y.; HILL, H. C. y BALL, D. L. (2011). Prospective teachers' learning to provide instructional explanations: How does it look and what might it take? Journal of Mathematics Teacher Education, 14 (6), 441-463.

CHICK, H. L. (2003). Pre-service teachers' explanations of two mathematical concepts. Documento presentado en Annual Conference of the Australian Association for Research in Education, Auckland, New Zealand.

CLIMENT, N. y CARRILLO, J. (2002). Developing and Researching professional Knowledge with Primary Teachers. En J. Novotna (Ed.), European Research in Mathematics Education II, vol. 1, 269-280. Praga: Charles University.

D'AMBROSIO, B. S. y MENDONCA-CAMPOS, T. N. (1992). Pre-service teachers' representations of children's understanding of mathematical concepts: Conflicts and conflict resolution. Educational Studies in Mathematics, 23, 213-230.

DOMONEY, B. (2001). Student teachers' understanding of rational numbers. En J. Winter (Ed.), Proceedings of the British Society for Research into Learning Mathematics, Vol. 21 (3) (pp. 13-18). Southampton: BSRLM.

FULLER, R. A. (1996). Elementary Teachers' Pedagogical Content Knowledge of Mathematics. Documento presentado en Mid-Western Educational Research Asociation Conference, Chicago, Illinois.

GAIRÍN, J. M. (2001). Sistemas de representación de números racionales positivos. Un estudio con maestros en formación. Contextos educativos, 4, 137-159.

GÓMEZ, P. (2006). Análisis didáctico en la formación inicial de profesores de Matemáticas de secundaria. En P. Bolea, M. J. González y M. Moreno (Eds.), X Simposio de la Sociedad Española de Investigación en Educación Matemática (pp. 15-35). Huesca, España: Instituto de Estudios Aragoneses.

GÓMEZ, P. (2007). Desarrollo del conocimiento didáctico en un plan de formación inicial de profesores de matemáticas de secundaria. Tesis doctoral no publicada, Universidad de Granada.

GÓMEZ, P. y GONZÁLEZ, M. J. (2013). Diseño de planes de formación de profesores de Matemáticas basados en el análisis didáctico. En L. Rico, J. L. Lupiáñez y M. Molina (Eds.), Análisis Didáctico en Educación Matemática, (pp. 121-139). Granada: Comares. 
GÓMEZ, P. y GUTIÉRREZ-GUTIÉRREZ, A. (2014). Conocimiento matematico y conocimiento didactico del futuro profesor espanol de primaria. Resultados del estudio TEDS-M. En M. T. Gonzalez, M. Codes, D. Arnau y T. Ortega (Eds.), Investigación en Educación Matemática (pp. 99-114). Salamanca: SEIEM.

HIEBERT, J. y CARPENTER, T. P. (1992). Learning and Teaching with Understanding. En D. A. Grouws (Ed.), Handbook of Research on Mathematics Teaching and Learning (pp. 65-97). New York: Macmillan.

HILL, H. C.; BALL, D. L. y SCHILLING, S. G. (2008). Unpacking pedagogical content knowledge: Conceptualizing and measuring teachers' topic-specific knowledge of students. Journal for Research in Mathematics Education, 39 (4), 372-400.

ISIKSAL, M. y CAKIROGLU, E. (2011). The nature of prospective mathematics teachers' pedagogical content knowledge: The case of multiplication of fractions. Journal of Mathematics Teacher Education, 14 (3), 213-230.

KINACH, B. (2002). Understanding and learning-to-explain by representing mathematics: epistemological dilemmas facing teacher educators in the secondary mathematics "methods" course. Journal of Mathematics Teacher Education, 5 (2), 153-186.

LI, Y. y KULM, G. (2008). Knowledge and confidence of pre-service mathematics teachers: the case of fraction división. ZDM, 40, 833-843.

LUPIÁÑEZ, J. L. y RICO, L. (2008). Análisis didáctico y formación inicial de profesores: competencias y capacidades en el aprendizaje de los escolares. PNA, 3 (1), 35-48.

MARKS, R. (1990). Pedagogical Content Knowledge: From a Mathematical Case to a Modified Conception. Journal of Teacher Education, 41 (3), 3-11.

NEWTON, K. J. (2008). An Extensive Analysis of Preservice Elementary Teachers' Knowledge of Fractions. American Educational Research Journal, 45 (4), 10801110.

PONTE, J. P. y CHAPMAN, O. (2008). Preservice mathematics teachers' knowledge and development. En L. D. English (Ed.), Handbook of international research in mathematics education (pp. 223-261). New York: Routledge.

RICO, L. y FERNÁNDEZ-CANO, A. (2013). Análisis didáctico y metodología de investigación. En L. Rico, J. L. Lupiañez y M. Molina (Eds.), Análisis Didáctico en Educación Matemática (pp. 1-22). Granada: Comares.

SHULMAN, L. S. (1986). Those who understand: Knowledge growth in teaching. Educational Researcher, 15 (2), 4-14.

SIMON, M. A. (1993). Prospective elementary teachers' knowledge of division. Journal for Research in Mathematics Education, 31, 5-25.

THANHEISER, E. (2009). Preservice elementary school teachers' conceptions of multidigit whole numbers. Journal for Research in Mathematics Education, 40, 252-281.

VALVERDE, G. (2012). Competencias Matemáticas promovidas desde la razón y la proporcionalidad en la formación inicial de maestros de educación primaria. Tesis doctoral no publicada, Universidad de Granada.

WEISS, I. R. y PARSLEY, J. D. (2004). What is high-quality instruction? Educational Leadership, 65 (1), 24-28. 